\title{
MILLIARCSECOND POLARIZATION PROPERTIES OF SEVERAL BL LACERTAE OBJECTS
}

\author{
D. C. Gabuzda, D. H. Roberts, J. F. C. Wardle, and L. F. Brown \\ Department of Physics \\ Brandeis University \\ Waltham, MA 02254 USA
}

\begin{abstract}
We discuss the $\lambda 6 \mathrm{~cm}$ total intensity and polarization structures of a number of BL Lacertae objects at milliarcsecond resolution. $0235+164$ was unresolved and weakly polarized at each of two epochs a year apart; each of the other objects displays structure in polarized flux. $0735+178$ and $1749+096$ can be adequately modeled by two or three point components-a "core" plus one or two "knots." The core components were moderately polarized $(\simeq 5 \%)$, while "knots" may be polarized at $8 \%$ or more, consistent with these components being optically thin. Preliminary results for BL Lac indicate that the total intensity structure can be modeled well by a set of four gaussian components; the polarization structure is complex, but is dominated by the northernmost knot in the jet.
\end{abstract}

\section{INTRODUCTION}

Here we report results of polarization-sensitive VLBI observations of the BL Lacertae objects $0235+164,0735+178,1749+096$, and $\mathrm{BL}$ Lac. Polarization-sensitive $\lambda 6 \mathrm{~cm}$ observations were carried out in December 1981 and December 1982 with the Haystack, Green Bank, and Owens Valley antennas and the phased-up VLA, and in March 1984 with the Bonn and Fort Davis antennas in addition to these four. In all cases the Mark III data recording system was used. Maps of the total intensity and of the distribution of polarized intensity were made as described by Roberts, Gabuzda, and Wardle (these proceedings).

\section{RESULTS}

We fit simple models to the visibilities produced by the hybrid mapping process and, when dynamic range permitted, required that the models account for the $I$ and $P$ data simultaneously. Maps of the $I$ and $P$ distributions of BL Lac are presented in Figure 1; maps of $0735+178$ can be found in Roberts and Wardle (1986). A more detailed discussion will be presented in Gabuzda, Roberts, and Wardle (1987).

Data for $0235+164$ were taken at the earlier two epochs. At the first epoch, the object is unresolved and polarized $\simeq 1.4 \%$ at position angle $39^{\circ}$. At the second epoch, model fitting renders some evidence for a secondary component at a separation of $\simeq 1.8$ mas from the core at position angle $45^{\circ}$, but the polarized fringes are too weak to determine the parameters of this component with any certainty. The core is $\simeq 1.2 \%$ polarized at position angle $\chi \simeq 110^{\circ}$, indicating that $\chi$ for the core rotated by at least $\simeq 70^{\circ}$ during the year between observations. 
Data for $0735+178$ were taken in December 1982. At this epoch, this object can be represented by an unresolved core and a jet-like extension at structural position angle $\simeq 69^{\circ}$ consisting of two knots, one located $\simeq 2.2$ mas from the core and one located $\simeq 4.7$ mas from the core. The inner knot has flux density $\simeq 200 \mathrm{mJy}$ and is $\simeq 3 \%$ polarized at position angle $\chi \simeq 20^{\circ}$; the outer knot has flux density $\simeq 160 \mathrm{mJy}$ and is $\simeq 8 \%$ polarized at $\chi \simeq 45^{\circ}$. The core is $\simeq 4 \%$ polarized at $\chi \simeq 4^{\circ}$. If the rotation measure for $0735+178$ is small, as is indicated in the data of Altschuler and Wardle (1976) and Aller et al. (1985), these polarization position angles indicate a $\mathbf{B}$ field which is predominantly parallel to the jet at the position of the core and rotates further down the jet until it is predominantly perpendicular to the jet at the position of the outer knot.

Data for $1749+096$ were taken in December 1981. The polarization structure can be represented by $a \simeq 2: 1$ double with a separation of $\simeq 0.8$ mas at position angle $\simeq 13^{\circ}$. Our dynamic range was insufficient to identify an $I$ counterpart to the northern $P$ component. The core is $\simeq 6.5 \%$ polarized at $\chi \simeq 40 ; \chi$ for the northern component is $\simeq 112^{\circ}$. If the rotation measure for this object is small, as is again indicated by the data of Altschuler and Wardle and of Aller et al., the B field direction inferred in the northern component is predominantly parallel to the position angle of the structure.

Data for BL Lac were taken in March 1984. Preliminary results indicate that the total intensity structure can be modeled by a set of four gaussian components-what may be a compact core plus three resolved knots in a jet which extends southward. The polarization structure has proved more difficult to model fit, but appears to be dominated by the northernmost knot.
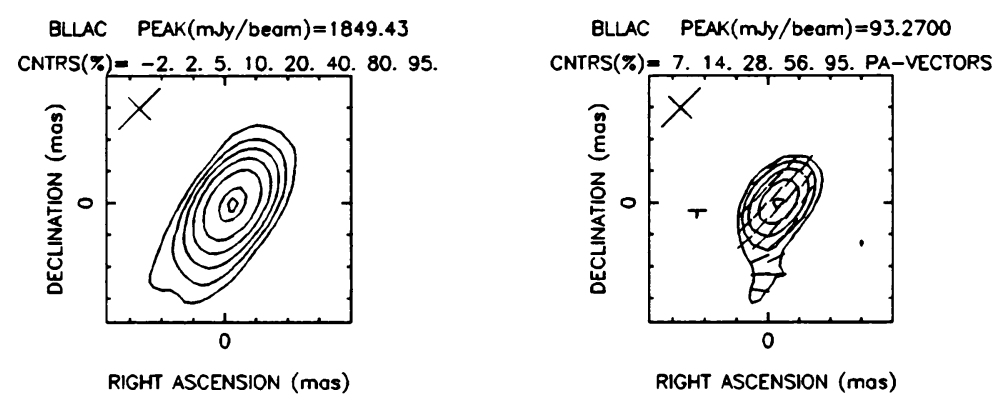

Figure 1. Hybrid $I$ and $P$ maps of BL Lac, March 1984 (1 tick mark equals 2 mas).

This work would not have been possible without the assistance of Dr. Alan Rogers. We also thank the staffs of the US VLBI Network observatories and of the Haystack Mark III Processor for their help. Financial support was provided by the NSF under grants AST82-13991 and AST-84-18636 (JFCW) and AST-83-15945 and AST-85-19529 (DHR).

\section{REFERENCES}

Aller, H. D., Aller, M. F., Latimer, G. E., and Hodge, P. E. 1985, Ap. J. Supp. 59, 513.

Altschuler, D. R., and Wardle, J. F. C. 1976, Mem. R. astr. Soc. 82, 1.

Gabuzda, D. C., Roberts, D. H., and Wardle, J. F. C. 1987, in preparation.

Roberts, D. H., et al. 1984, in VLBI and Compact Radio Sources, ed. R. Fanti, K. Kellermann, and G. Setti (Dordrecht: Reidel), 35.

Roberts, D. H., and Wardle, J. F. C. 1986, in Quasars, ed. G. Swarup and V. K. Kapahi, (Dordrecht: Reidel), 141. 